\title{
Dynamic Characteristics of the Opto-Fluidic Conversion Device
}

\author{
Fumitaka KIKKAWA*, Osamu OYAMA*, \\ Toshihiro YOSHIMITSU** and Keijiro YAMAMOTO** \\ * Graduate School of Science and Technology \\ Meiji University \\ 1-1-1, Higashimita, Tama-ku, Kawasaki-shi, Kanagawa, 214-8571 Japan \\ (E-mail: ce55220@isc.meiji.ac.jp) \\ ** Welfare Systems Engineering \\ Kanagawa Institute of Technology \\ 1030, Shimo-ogino, Atsugi-shi, Kanagawa, 243-0292 Japan
}

\begin{abstract}
This report treats a development of an opto-fluidic conversion device. Using a bundled optical fiber which has the sectional area of $1.0[\mathrm{~mm}] \times 5.0[\mathrm{~mm}], 2.0[\mathrm{~mm}] \times 5.0[\mathrm{~mm}]$ and $3.0[\mathrm{~mm}] \times 5.0[\mathrm{~mm}]$ respectively, the dynamic characteristics on an opto-fluidic conversion device have been analyzed. This paper reports analysis result of the dynamic characteristics and the effect on the size of the optimal sectional area of the bundled optical fiber. From the analytical result of the dynamic characteristics, it has been confirmed that the bundled optical fiber with the sectional area of $2.0[\mathrm{~mm}] \times 5.0[\mathrm{~mm}]$ has been optimal in the test case.
\end{abstract}

\section{Key Words}

Opto-fluidic converter, Optical control, Laminar Proportional Amplifier, Optical fiber, Output differential pressure

\section{Introduction}

Electric equipment is used in a wide field now, and embedded in a majority of machines. This equipment operates on an electric signal. However, there is a possibility which may not operate correctly in an environment where electromagnetic noises or radioactivities exist in.

An opto-fluidic control system converts an optical signal into a pneumatic signal directly without an intermediate electric signal, and realizes a control of machine without electric device[1]. Therefore, this system enables to be used in the severe environment like above-mentioned. An opto-fluidic converter which is the peculiar feature of this system is composed of an opto-fluidic conversion device and a Laminar
Proportional Amplifier (LPA) of two stages. The LPA is a kind of fluidic amplifier. The opto-fluidic conversion device, which is the first stage of this converter, has a shape based on the LPA. Concretely, two control ports are removed from the inside of LPA, and optical fibers affixed with optical absorber at the fiber end are embedded there. The optical signal is converted into the pneumatic signal by this conversion device, and the converted pneumatic signal is amplified by the LPA of two stages.

We have analyzed the dynamic characteristics of fluid in this opto-fluidic conversion device. In analyzing the dynamic characteristics, there is a problem that the device was too small with the original size arose. 
Considering the size of the measuring instrument, it was difficult to exactly analyze the dynamic characteristics. Therefore, an expansion model which is expanded the original device to double height and width size was made. In order to correspond to the expansion of the device, the bundle of the optical fiber was made. The shape of bundled optical fiber is rectangular pillar and the sectional area of which is $2.0[\mathrm{~mm}] \times 5.0[\mathrm{~mm}]$ [2].

In the present study, we made three kinds of the bundled optical fiber newly, i.e., the sectional area of which are $1.0[\mathrm{~mm}] \times 5.0[\mathrm{~mm}], 2.0[\mathrm{~mm}] \times 5.0[\mathrm{~mm}]$ and $3.0[\mathrm{~mm}] \times 5.0[\mathrm{~mm}]$ respectively. We have investigated the dynamic characteristics of these bundled optical fibers.

This paper is intended to report the optimal sectional area of the bundled optical fiber which we have selected through the experiments on the dynamic characteristics.

\section{Principle of operation and structure}

\subsection{Laminar Proportional Amplifier (LPA)}

Figure 1 shows the structure of the LPA, which has symmetrical structure. The principle of operation of the LPA is as follows:

(1) The supply jet is inputted form the supply port, and the differential pressure is inputted from right and left of two control ports which exist inside the LPA.

(2) In proportion to this differential pressure, a part of supply jet leans; for example, assuming that the pressure from the control port $\mathrm{A}$ is higher than that from the control port $\mathrm{B}$, the part of supply jet leans toward the opposite direction in which the output port B exists.

(3) By leaning the part of the supply jet, a difference arises between the pressures outputted from two output ports.

The LPA enables to amplify the feeble differential pressure, because the laminar jet is used as the supply jet of the LPA. The LPA is attached to the output port of opto-fluidic conversion device in order to amplify the feeble differential pressure from the conversion device.

\subsection{Opto-fluidic conversion device}

The opto-fluidic conversion device is made from the LPA which is a basic element. Figure 2 shows the structure of this conversion device. The main difference is that the conversion device has not control ports. And the optical fibers are embedded in the position of control ports. In these fibers, the optical absorber is affixed at the fiber end. The principle of operation of this device is as follows:

(1) When light from laser source is irradiated the optical fiber, this light passes through the optical fiber, and reaches the optical absorber affixed at the fiber end.

(2) The optical absorber, which is a carbon black, has a character to convert light into heat. Therefore, light which reaches at the fiber end is converted into heat by the optical absorber.

(3) Generated heat causes partially the temperature change of the supply jet at the vicinity of fiber end. As a result, viscosity of the supply jet rises, because viscosity of gas rises along with the temperature rise.

(4) By the viscosity rise, a flow profile of jet becomes asymmetrical for the nozzle axis, and a difference is generated in the flow velocity of jet for the nozzle axis. Therefore, the output differential pressure proportional to the intensity of light is generated from the output port $A$ and $B$.

Thus, this conversion device enables to convert an optical signal into a corresponding pneumatic signal without mechanical drive components.

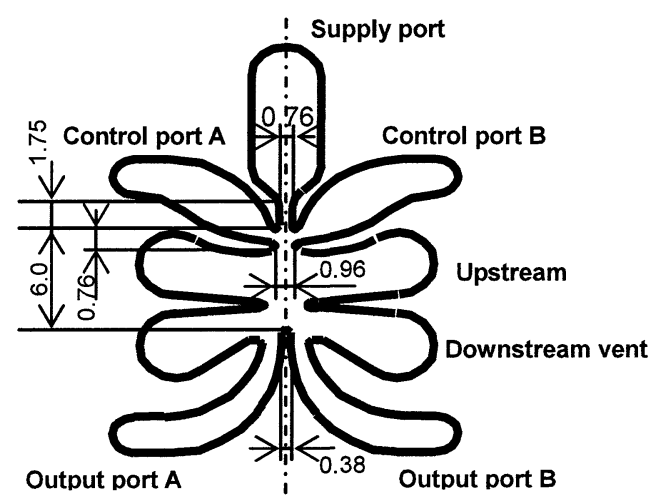

Fig.1 Structure of Laminar Proportional Amplifier

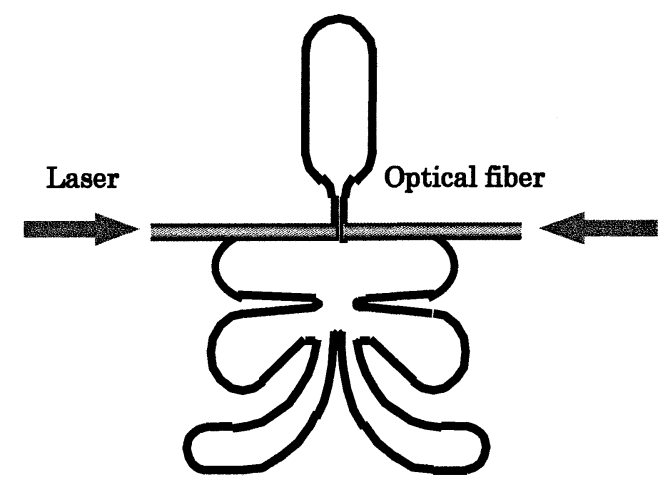

Fig.2 Structure of opto-fluidic conversion device 


\subsection{Opto-fluidic converter}

Figure 3 shows the structure of the opto-fluidic converter. The 1st stage of this converter is the opto-fluidic conversion device. The output differential pressure generated by the conversion device is feeble. Therefore, it is amplified fluidically by the LPA of two stages which are 2nd and 3rd stage. The aspect ratio is 1.32 in both the 1 st conversion stage and the 2nd stage, and that of $3 \mathrm{rd}$ stage is 0.66 . The thickness of this converter is $0.5[\mathrm{~mm}]$. We have analyzed the dynamic characteristics of the opto-fluidic conversion device which is 1st stage. In our experiments, we have not used this opto-fluidic converter itself but the expansion model.

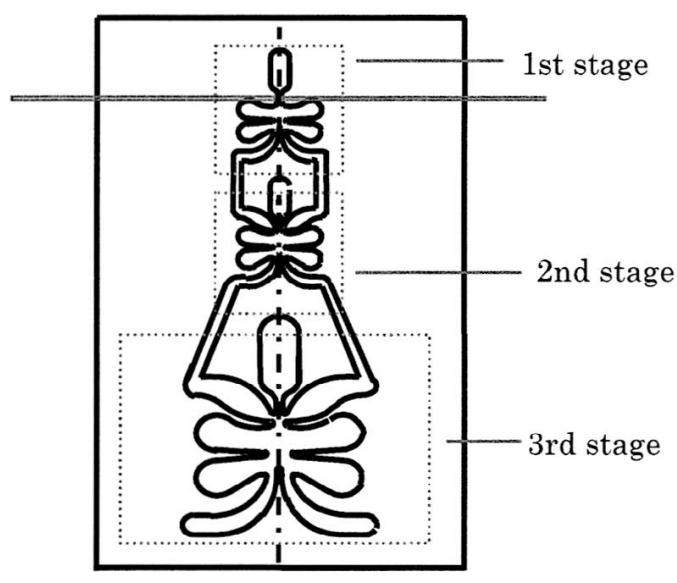

Fig.3 Structure of opto-fluidic converter

\section{Experiment instrument}

\subsection{Expansion model}

In analyzing the dynamic characteristics of opto-fluidic conversion device, considering the size of the measuring instrument, it was difficult to analyze the dynamic characteristics by using the conversion device of original size, because the conversion device is too small compared with the size of measuring instrument. Therefore, we make a model, which is expanded the conversion device of original size to double height and width size. Figure 4 shows the structure of this expansion model. It is made of stainless steel, and the thickness is $5.0[\mathrm{~mm}]$.

\subsection{Bundled optical fiber}

As the optical fiber for this model expanded to double height and width size, the bundle of optical fiber is used. The sectional area is $2.0[\mathrm{~mm}] \times 5.0[\mathrm{~mm}]$, and the shape of the bundled optical fiber is rectangular pillar. In addition, we make more two kinds of the bundled optical fiber, the sectional area of which is $1.0[\mathrm{~mm}] \times 5.0[\mathrm{~mm}]$ and $3.0[\mathrm{~mm}] \times 5.0[\mathrm{~mm}]$ respectively. The shape of those fibers is also rectangular pillar. Figure 5 shows the configuration of bundled optical fiber.
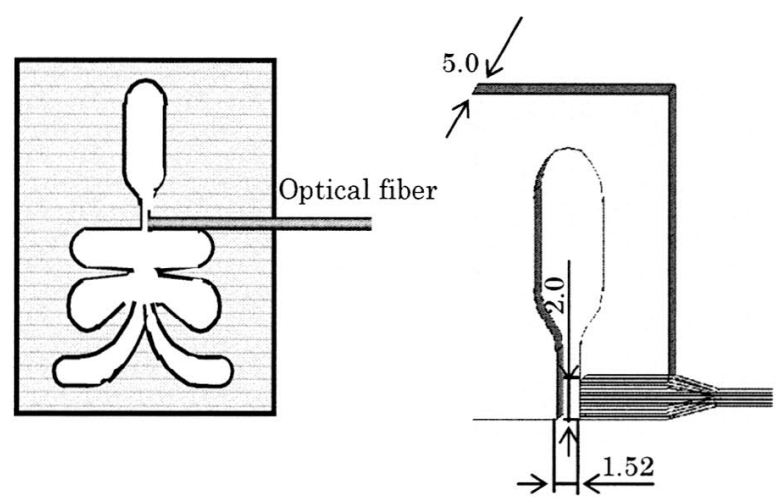

Fig.4 Structure of expansion model
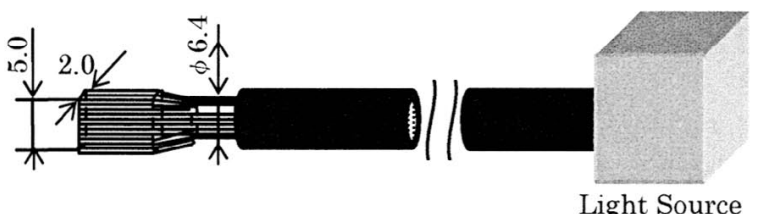

Fig.5 Configuration of bundled optical fiber

\section{Experimental results}

Three kinds of experiments on the dynamic characteristics are conducted by using the expansion model. The optimal sectional area of the three bundled optical fibers is examined from these experiments.

\subsection{Temperature measurement of the optical absorber}

The temperature change of the optical absorber influences the air flow in the opto-fluidic conversion device. Therefore, the time change of the temperature on the optical absorber is measured by using the expansion model and bundled optical fibers. The sectional area of bundled optical fibers are $1.0[\mathrm{~mm}] \times 5.0[\mathrm{~mm}], 2.0[\mathrm{~mm}]$ $\times 5.0[\mathrm{~mm}]$ and $3.0[\mathrm{~mm}] \times 5.0[\mathrm{~mm}]$. Figure 6 and Figure 7 show the measurement result concerning three bundled optical fibers. The result is shown by the approximation curve. Where, the supply pressure to the conversion device is $100[\mathrm{~Pa}]$, and the temperature of the fiber end is unified as $100\left[{ }^{\circ} \mathrm{C}\right]$ in these bundled optical fibers. The 
time when the light is begun to irradiate is set to $0[\mathrm{~s}]$ in Fig.6, and the time when the light disappears is set to 0 ¡s〉 in Fig.7.

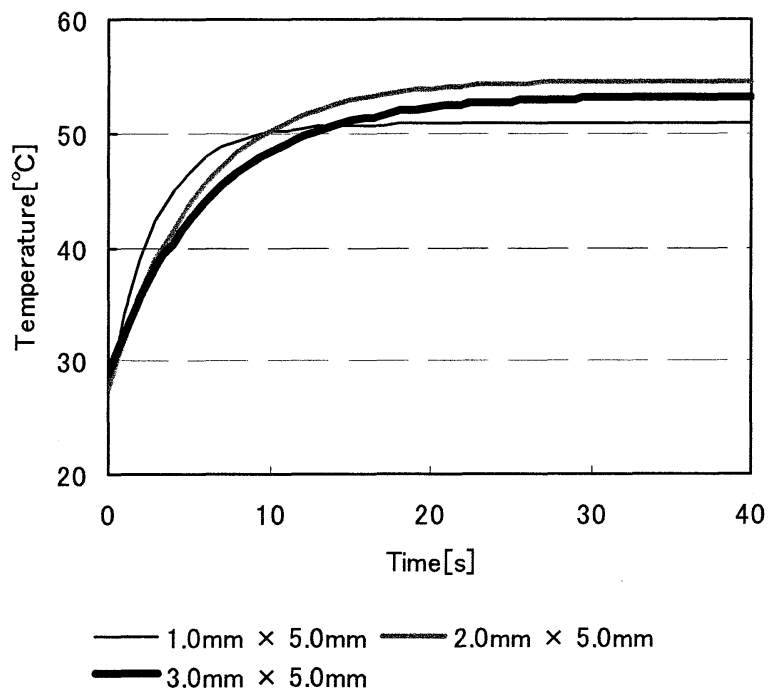

Fig.6 Temperature of the optical absorber, case of rise

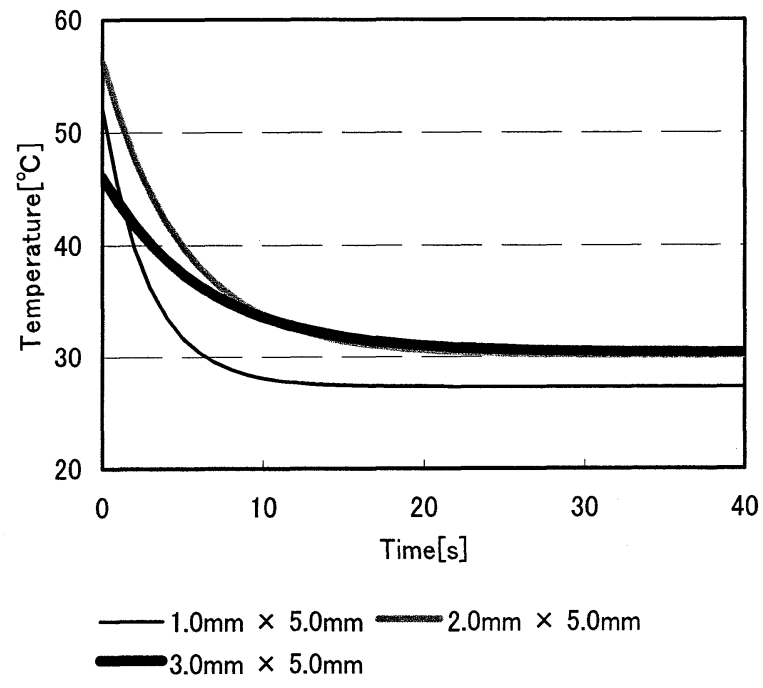

Fig.7 Temperature of the optical absorber, case of descent

From Fig.6 and Fig.7, it is shown that the time of transient response is proportional to the size of the sectional area of the bundled optical fiber. The difference of transient response time more than $10[\mathrm{~s}]$ appears between the result of bundled optical fiber of $1.0[\mathrm{~mm}] \times$ $5.0[\mathrm{~mm}]$ and that of $3.0[\mathrm{~mm}] \times 5.0[\mathrm{~mm}]$. Therefore, we consider that an area of the optical absorber influences the time constant of a first order lag system.

\subsection{Measurement of the output differential pressure}

In this experiment, the output differential pressure from the opto-fluidic conversion device is measured. Figure 8 shows the measurement result of the output differential pressure concerning three bundled optical fibers. The sectional area of these fibers are $1.0[\mathrm{~mm}] \times 5.0[\mathrm{~mm}]$, $2.0[\mathrm{~mm}] \times 5.0[\mathrm{~mm}]$ and $3.0[\mathrm{~mm}] \times 5.0[\mathrm{~mm}]$. The result is shown by the approximation curve. Where, the supply pressure to the conversion device is $100[\mathrm{~Pa}]$, and the temperature of the fiber end is unified as $100\left[{ }^{\circ} \mathrm{C}\right]$ in these bundled optical fibers. The time when light is irradiated is set to $0[\mathrm{~s}]$, too.

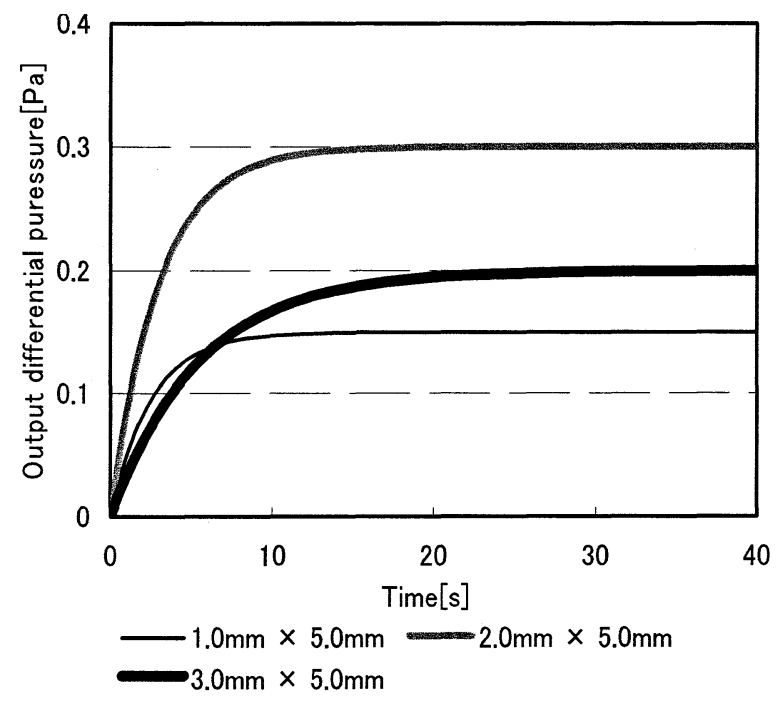

Fig.8 Output from the opto-fluidic conversion device

From Fig.8, it is shown that the sectional area of bundled optical fibers is not proportional to the output differential pressure from the conversion device. The bundled optical fiber of $1.0[\mathrm{~mm}] \times 5.0[\mathrm{~mm}]$ doesn't generate the viscosity change effectively because the quantity of heat which gives to the supply jet is a little. By contrast, the bundled optical fiber of $3.0[\mathrm{~mm}] \times 5.0$ $[\mathrm{mm}]$ generates the viscosity change in the large range because there is much quantity of heat given to the supply jet. Therefore, it is considered that the output differential pressure is low when the bundled optical fiber of $1.0[\mathrm{~mm}] \times 5.0[\mathrm{~mm}]$ or $3.0[\mathrm{~mm}] \times 5.0[\mathrm{~mm}]$ is used. We consider that the bundled optical fiber of 2.0 $[\mathrm{mm}] \times 5.0[\mathrm{~mm}]$ enables to obtain the highest output differential pressure.

\subsection{Measurement of the pneumatic temperature change in the opto-fluidic conversion device}

In this experiment, the time change of the pneumatic temperature in the opto-fluidic conversion device is 
measured by using the expansion model. As shown in Fig.9, measurement positions are three places, where are $0.05[\mathrm{~mm}]$ (Region 1), 5.0[mm] (Region 2) and $12.0[\mathrm{~mm}]$ (Region 3) downward from a nozzle edge. The supply pressure to the conversion device is $100[\mathrm{~Pa}]$, and the temperature of the fiber end is unified as $100\left[{ }^{\circ} \mathrm{C}\right]$ in three bundled optical fibers, the sectional area of which are $1.0[\mathrm{~mm}] \times 5.0[\mathrm{~mm}], 2.0[\mathrm{~mm}] \times 5.0[\mathrm{~mm}]$ and $3.0[\mathrm{~mm}] \times$ $5.0[\mathrm{~mm}]$. Figure 10.1 and 10.2 show the temperature distribution of the Region 1 in the conversion device, and figure 11.1 and 11.2 show that of the Region 3 in the conversion device. The temperature distribution are two kinds of $3[\mathrm{~s}]$ and $30[\mathrm{~s}]$ later when the light is irradiated. Figure 10.1 shows the temperature distribution of $3[\mathrm{~s}]$ later, and figure 10.2 shows that of 30 [s] later. Figure 11.1 shows the temperature distribution of $3[\mathrm{~s}]$ later, and figure 11.2 shows that of $30[\mathrm{~s}]$ later.

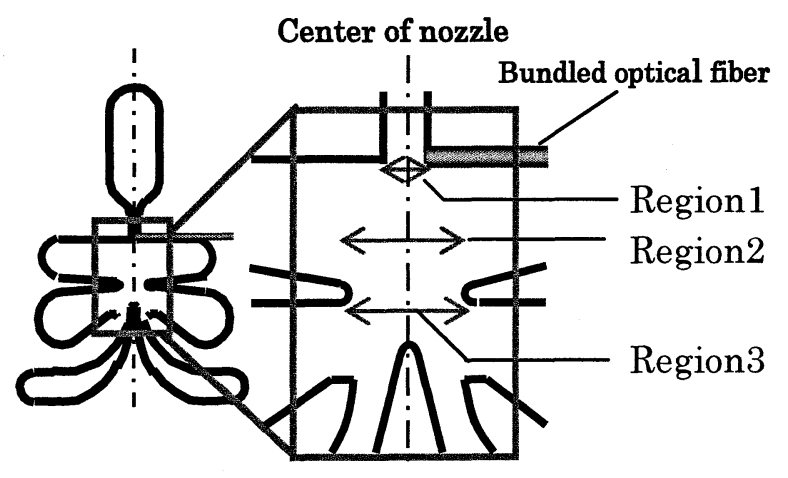

Fig.9 Measurement positions

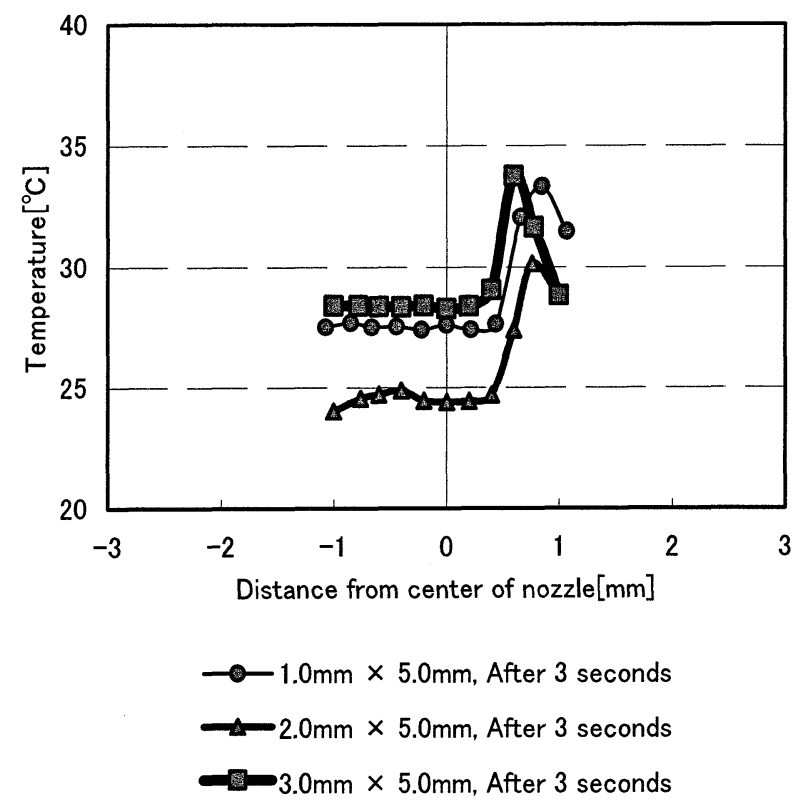

Fig.10.1 Temperature change in Region1(after 3 seconds)
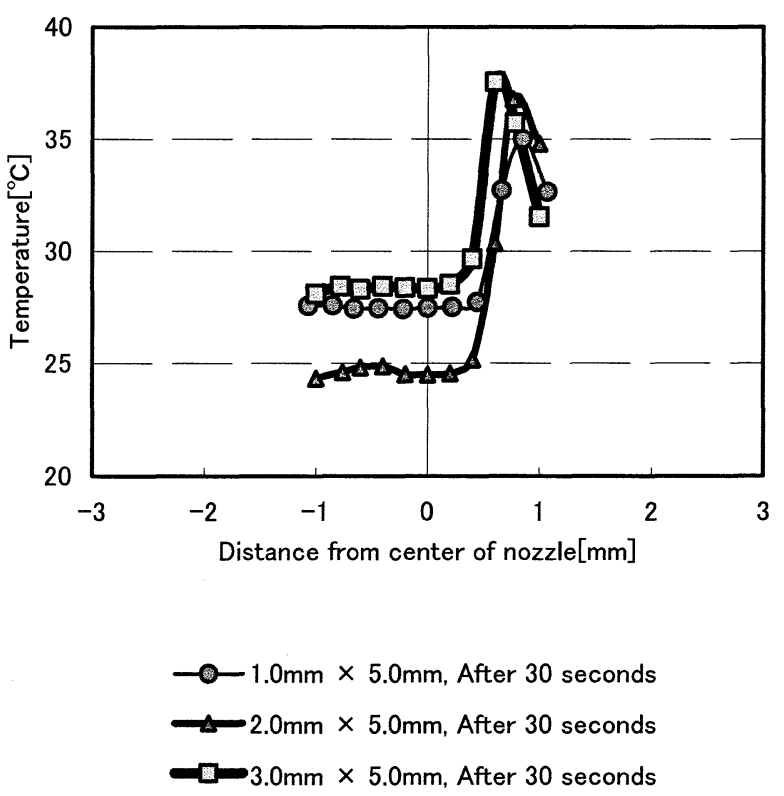

Fig.10.2 Temperature change in Region1(after 30 seconds)

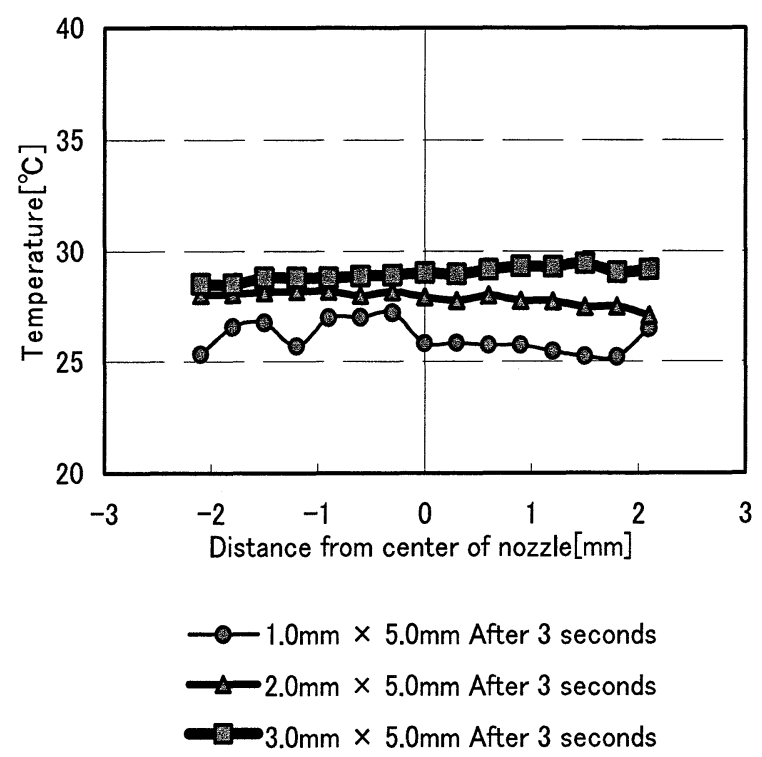

Fig.11.1 Temperature change in Region3(after 3 seconds) 


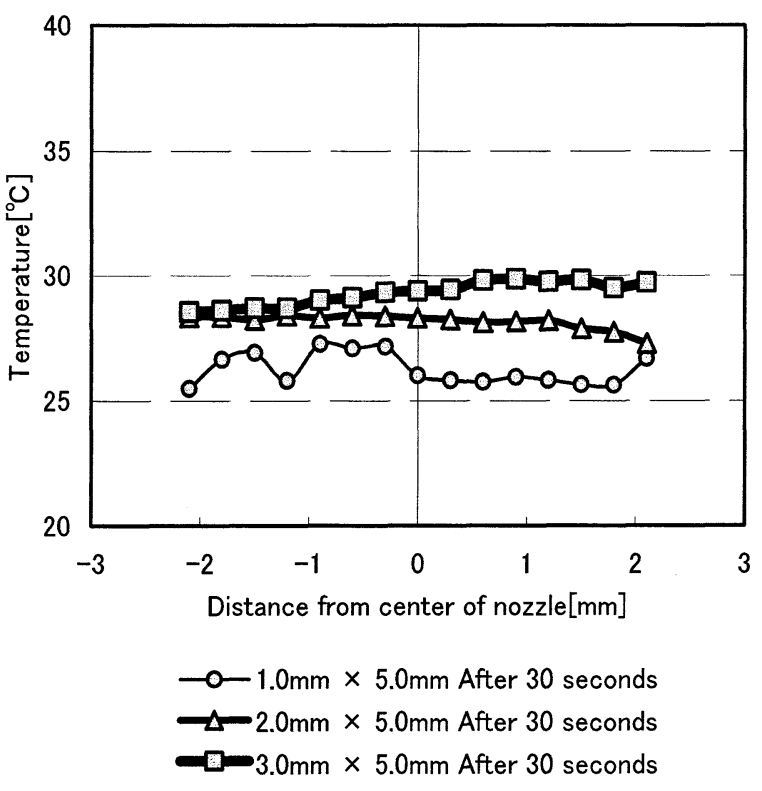

Fig.11.2 Temperature change in Region3(after 30 seconds)

From Fig.10.1, Fig.10.2, Fig.11.1 and Fig11.2, it is shown that the temperature change of the Region 1 is larger than that of the Region 3 in all bundled optical fibers. The temperature change becomes small as the measurement position moves to downward (i.e. from Region 1 to Region 3). It is considered that the temperature change of Region 1 is the largest compared with other measurement position because the Region 1 is the nearest measurement position from the bundled optical fiber. In Region 1 and Region 3, the temperature in the conversion device becomes the highest when the bundled optical fiber which is $3.0[\mathrm{~mm}] \times 5.0[\mathrm{~mm}]$ is used.

\section{Conclusion}

In the present study, three kinds of bundled optical fibers have been made, which have the different sectional area. And three kinds of the dynamic characteristics on the opto-fluidic conversion device have been measured by using those fibers. Measurement results have indicated that the size of the sectional area of the bundled optical fiber has greatly influenced the output differential pressure from the conversion device and the pneumatic temperature in it. In addition, it has indicated that the quantity of heat generated on the optical absorber has been proportional to the size of the sectional area of the fiber. It is notable that the output differential pressure is low when the sectional area of the fiber is too large or is too small. We consider that the bundled optical fiber which has the sectional of $2.0[\mathrm{~mm}] \times 5.0[\mathrm{~mm}]$ is optimal in this test case. If the performance of the actual opto-fluidic conversion device is improved referring these data, the opto-fluidic control system advances for the practical use.

\section{References}

1. Keijiro Yamamoto, Characteristics of an Opto-Fluidic Converter Utilizing Photo acoustic Effect, Trans. SISE Japan, 1991, 27-12, pp1405-1411.

2. Tomoaki Kurihara, Osamu Oyama, Toshihiro Yoshimitsu and Keijiro Yamamoto, Dynamic Characteristics of Opto-Fluidic Conversion Device, SICE 5th Symposium on Fluid Control and Measurement. 